\title{
The Pillow Project: A low cost intervention with the potential for widespread application to enable training in the event of an infant choking and basic life support for prospective parents

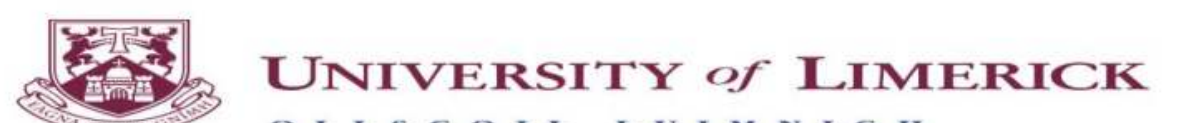

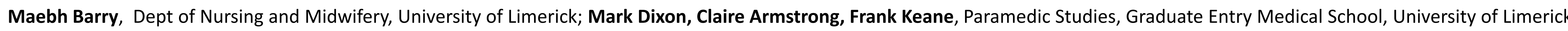

Background: Infant basic life support skills are not taught routinely to prospective and new parents across Europe and the United states. Very little research has been conducted on pregnant women and the provision of paediatric basic life support. Limited previous research has reported positive findings (Barry, 2015)*.

Aim: This study aimed to evaluate the impact of a self-instruction infant CPR kit on expectant parents. The kit included access to an e-learning training video and a novel low-cost infant pillow mannequin.

Design: A prospective cohort study with an uncontrolled pre- and posttest design was used following institutional ethical approval.

Setting: A four week cycle of antenatal education classes in a regional Maternity Hospital in Ireland.

Participants: A purposive sample of pregnant women and their partners attending the antenatal education classes.

Seed funding: EHS Faculty, University of Limerick

\section{Contact details}

Maebh Barry, maebh.barry@ul.ie; 061233653

Mark Dixon, mark.dixon@ul.ie, 061202941

Claire Armstrong, claire.armstrong@ul.ie, 061213337 rank Keane, frank.keane@ul.ie, 061234931
The pillow mannequin is a pillowslip designed to fit any standard sized pillow.

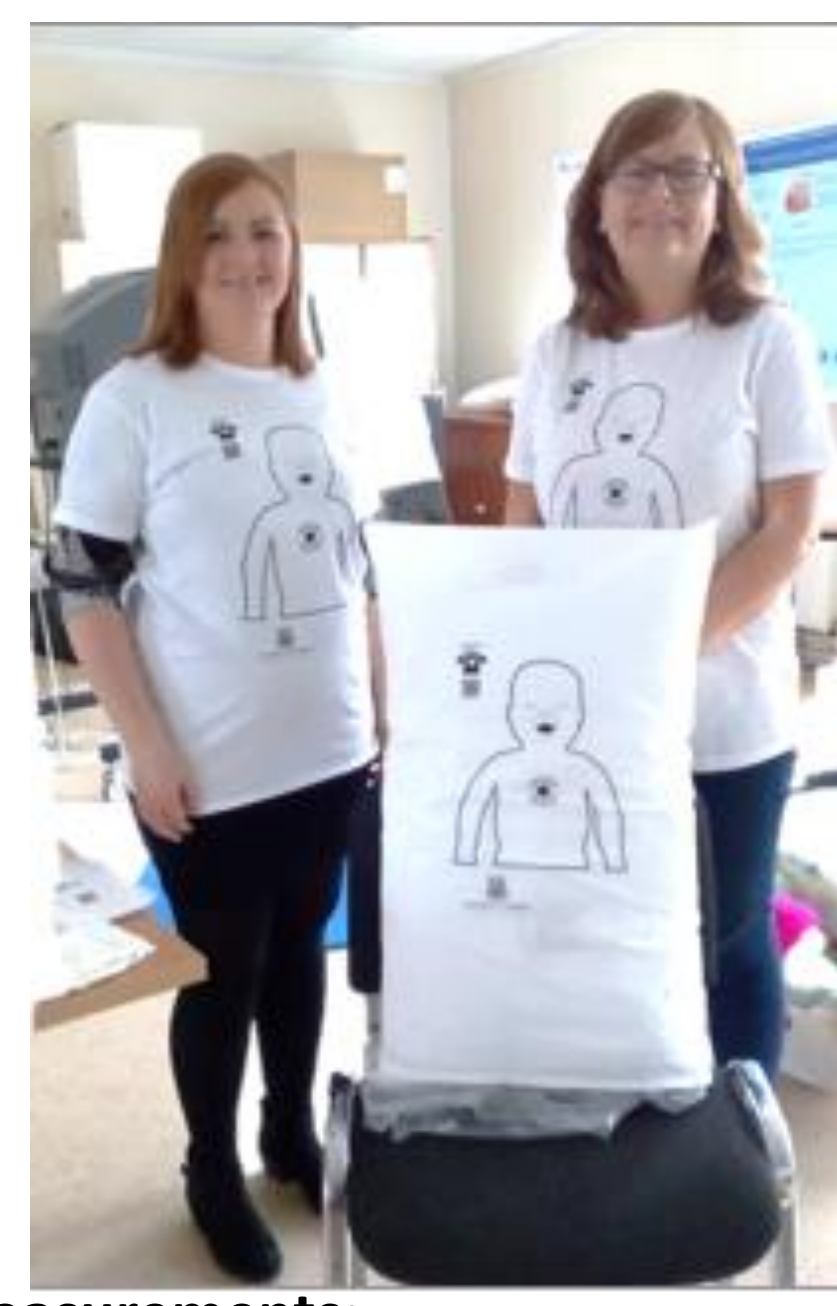

Measurements:

(1) confidence in performing infant CPR

(2) confidence in knowledge of infant choking treatment

(3) ability to perform requisite skills, and

(4) perceived acceptability of the infant pillow mannequin as a means of practise.

Results:

(1) Greater confidence in infant CPR post-training

(2) Greater confidence in infant choking treatment post-training

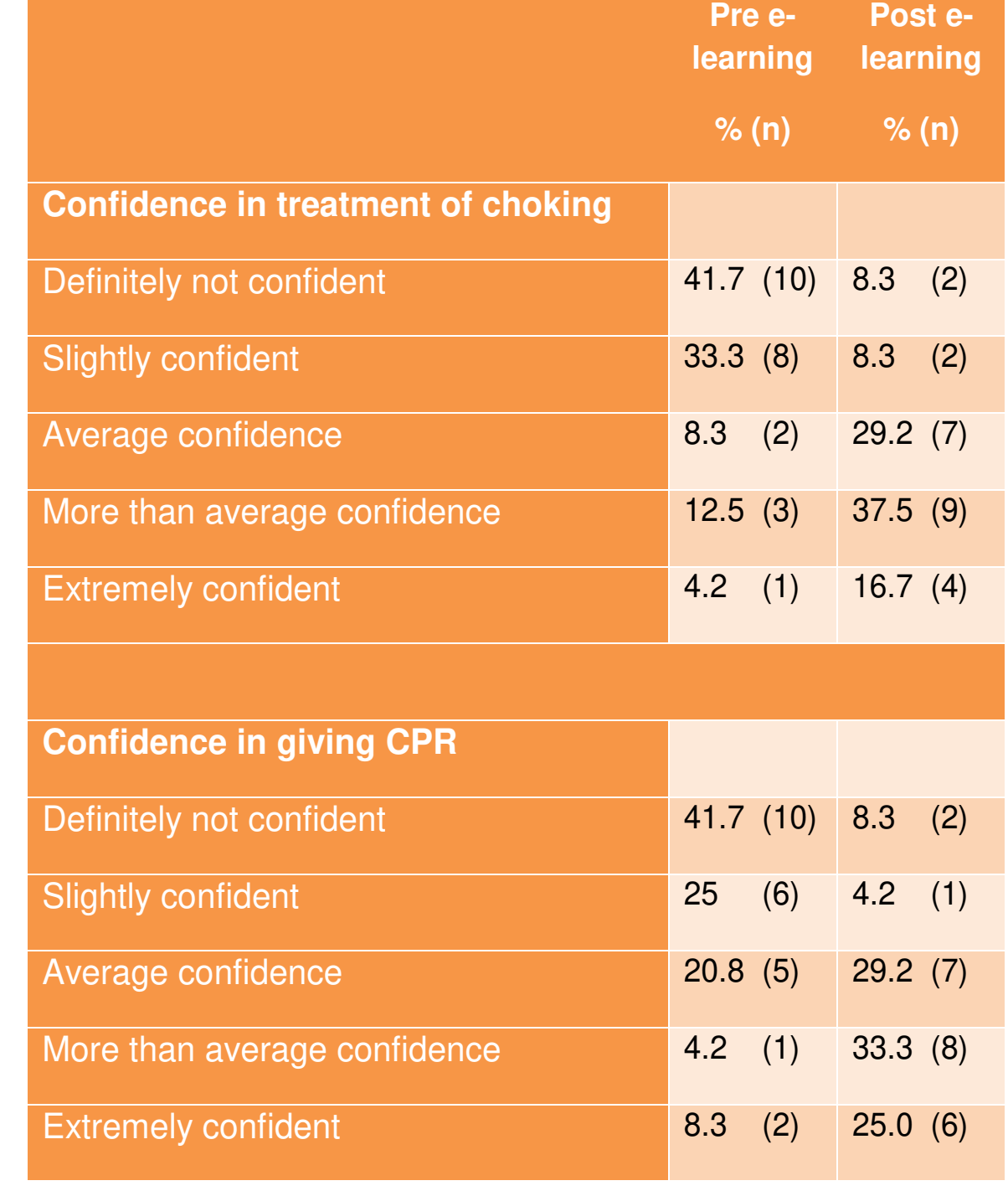

Table 1: Confidence pre and post e-learning programme

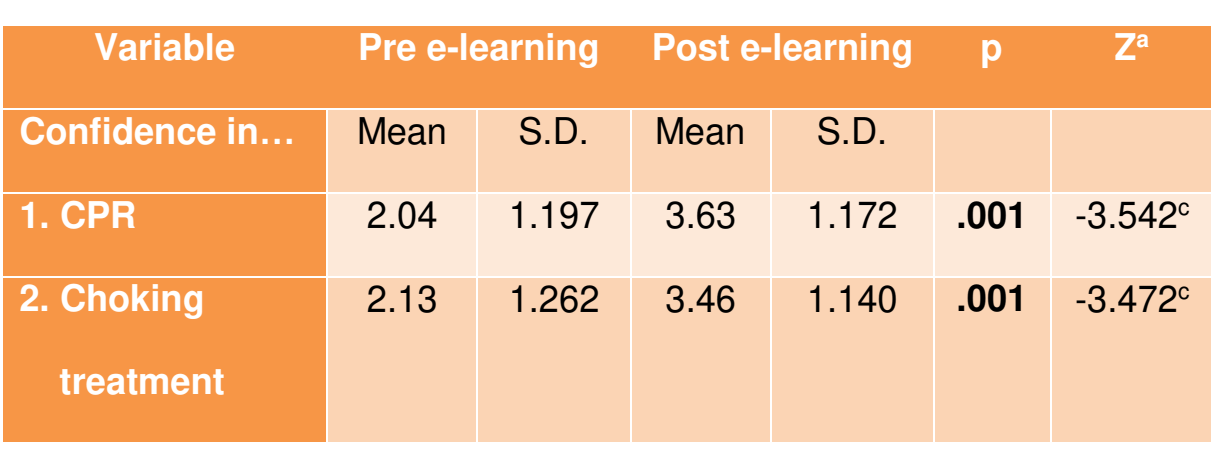

Table 2: Comparison of 'confidence' means and S.D. pre and post intervention

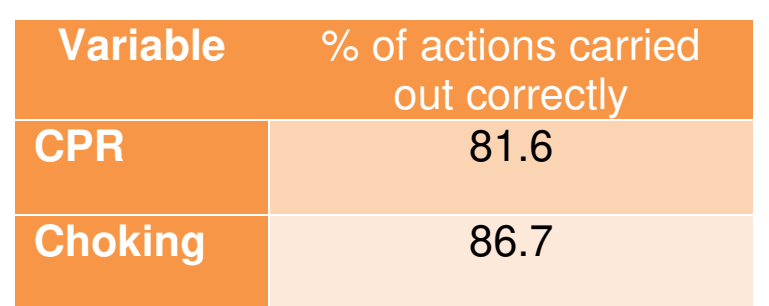

(4) The pillow mannequin was reported by expectant parents as being highly acceptable to them.

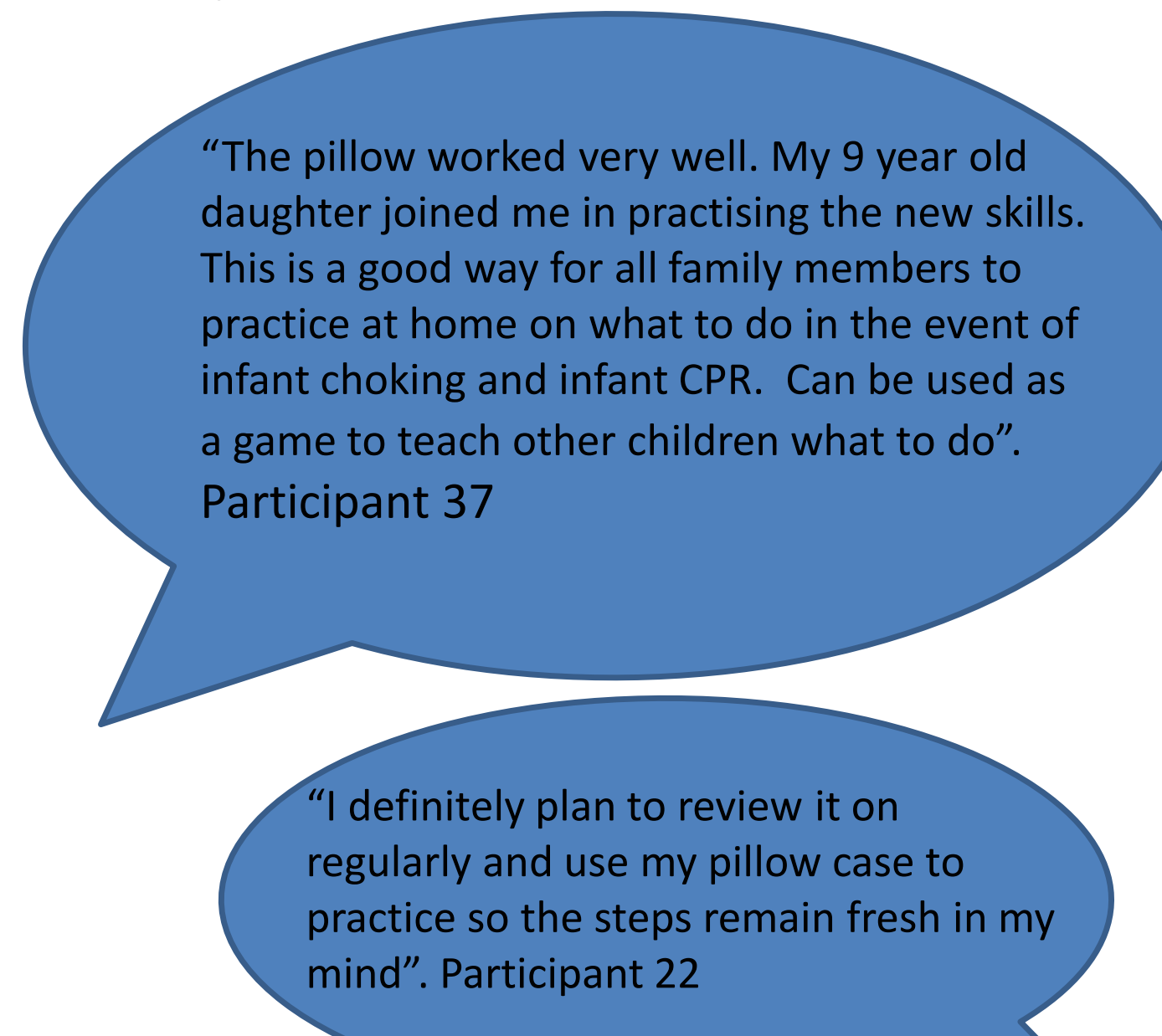

Discussion and conclusion

Results indicated that this was an acceptable and effective medium for training expectant parents in infant CPR and choking treatment.

The particular value of this training programme lies in the fact that the cost of both the e-learning training programme and infant pillow mannequin is very low, affording the potential for its widespread distribution to both developed and developing countries. 